\title{
Cryo-Electrical Microscopy for Quantum and Advanced Energy Applications
}

\author{
Khim Karki, Daan Hein Alsem and Norman Salmon
}

Hummingbird Scientific, Lacey, Washington, United States

The introduction of cryogenic cooling of specimens in the (scanning) transmission electron microscopy (S/TEM) has allowed a growing understanding of various quantum interfaces and phase co-existence in strongly correlated systems [1]. Understanding those quantum properties at the fundamental level has previously been challenging due to limited characterization techniques with inadequate spatial and temporal resolution. Quantum materials must be studied at cryogenic temperatures because many of the relevant properties in these quantum materials only manifest themselves at these low temperatures. Cryogenic S/TEM has also led to the observation of critical structural information related to battery interfaces at atomic resolution, which are traditionally difficult to achieve because they are air-sensitive and prone to electron beam damage at room temperature [2-5]. Thus, most materials require cryo-transfer and cryo-preservation of the sample using cryogenic coolant (liquid nitrogen), limiting the sample's studying to post-mortem analysis. Current relevant studies focused on quantum material were limited to post-mortem imaging at a cryogenic temperature only [5]. The capability did not focus on in-situ measuring the electrical transport properties of materials at cryogenic temperatures. Here, we present the development cryo-electrical biasing S/TEM holder that simultaneously allows electrical stimulus and high-resolution imaging of a sample in-situ at various cryogenic temperatures.

The newly developed cryo-biasing TEM holder will enable the understanding of critical interfaces in battery systems and surface-electronic behavior of quantum topological insulators such in twodimensional (2D) materials, among others, for artifact-free evaluation of the studied devices in highresolution imaging and spectroscopy modes. Here, we present an example of studying battery processes using a single nanowire system from room temperature down to liquid nitrogen temperature as close as around $-170^{\circ} \mathrm{C}$ [6]. Electrical biasing is performed in a nanowire sample that sits across the electrodes on the biasing chip (Figure 1). A constant current experiment at cold temperatures on the nanowire shows a voltage drop as the reaction proceeds with a growth of the dendrite layer plated on the nanowire's surface. The cryo-biasing TEM holder will be vital in enabling scientists to expand the knowledge of structureproperty relationships in materials, specifically the relation between temperature and electronic properties, and will allow for the accelerated development of the next generation of electronic, quantum, and energy storage materials devices [7]. 
A
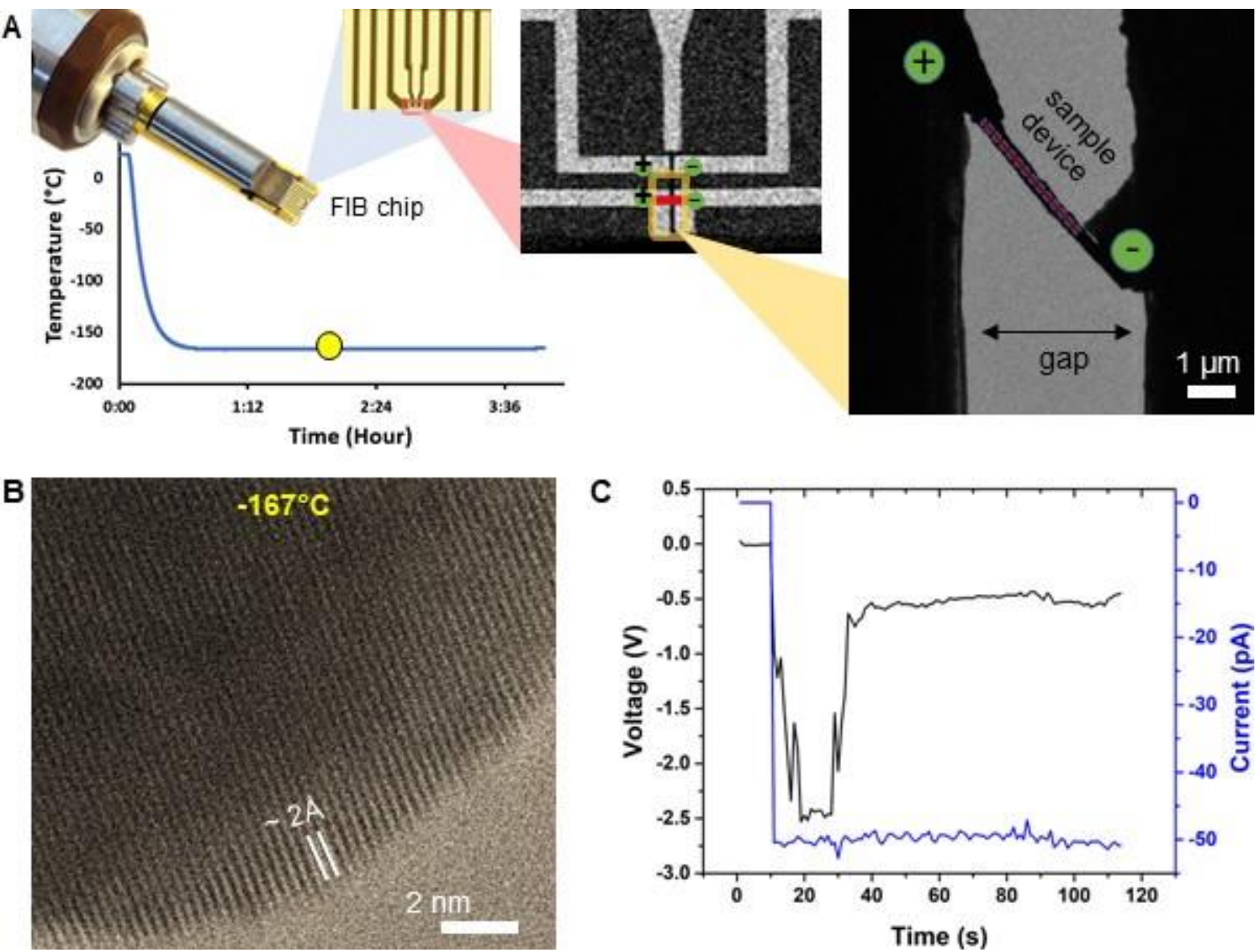

Figure 1. Figure 1. (A) Hummingbird Scientific Cryo Biasing TEM holder. The holder cools the sample from the room temperature down to $\sim-170^{\circ} \mathrm{C}$ and has a stable life of 4 hours at the lowest operating temperature. The holder allows on-chip biasing with up to 9 electrical contacts. The inset shows the higher magnification picture of the biasing chip with a gap (vacuum) across the pre-patterned metal leads. The sample device, in this case, a battery nanowire, is deposited across the positive $(+)$ and negative (-) leads for electrical transport measurements and cycling. (B) A high-resolution TEM image (HRTEM) showing resolved lattices of gold $(\mathrm{Au})$ nanocrystals at $-167^{\circ} \mathrm{C}$. The image is taken at the yellow dot region of the temperature profile shown in Figure $A$. (C) Current-voltage (I-V) characteristic as measured across a nanowire (Figure A) with a current in the pA range [6]. A constant current experiment on the nanowire in the Cryo-Biasing holder showing voltage drop as the reaction proceeds.

\section{References}

[1] K. A. Moler, Nat. Mater. 2017, 16, 1049.

[2] Y. Li, et al., Science (80-. ). 2017, 358, 506 LP.

[3] X. Wang, et al., Nano Lett. 2017, 17, 7606.

[4] M. J. Zachman, et al., Nature 2018, 560, 345.

[5] K.A. Spoth, et al., Microsc. Microanal (Suppl 2) 2019, 1660-1661

[6] Research material and image courtesy of UCSD/Battery500 Consortium

[7] KK, DHA, and NS acknowledge funding from the Department of Energy, Office of Basic Energy Sciences, SBIR Grant \# DE-SC0019627. 